\title{
Individualized Exercise and Diet Recommendations: An Expert System for Monitoring Physical Activity and Lifestyle Interventions in Obesity
}

\author{
Yunyoung $\mathrm{Nam}^{\dagger}$ and Yeesock Kim*
}

\begin{abstract}
This paper proposes an exercise recommendation system for treating obesity that provides systematic recommendations for exercise and diet. Five body indices are considered as indicators for recommend exercise and diet. The system also informs users of prohibited foods using health data including blood pressure, blood sugar, and total cholesterol. To maximize the utility of the system, it displays recommendations for both indoor and outdoor activities. The system is equipped with multimode sensors, including a three-axis accelerometer, a laser, a pressure sensor, and a wrist-mounted sensor. To demonstrate the effectiveness of the system, field tests are carried out with three participants over 20 days, which show that the proposed system is effective in treating obesity.
\end{abstract}

Keywords: Health care, Service recommendation, Multimodal sensors, Obesity, Multiple sensors

\section{Introduction}

The rapid recent increase in the global prevalence of obesity has been characterized as an epidemic by the World Health Organization (WHO) [1]. In most affluent populations, the prevalence of obesity among adults is 10 $40 \%$. Being overweight affects an even larger proportion of the population than obesity; approximately $65 \%$ of United States adults are currently overweight or obese, and the number of overweight adolescents in the United States has tripled in the past 20 years $[2,3]$ due to poor dietary habits and a lack of physical activity [4]. Diet and physical inactivity are the two main modifiable determinants of obesity. As reported by the WHO, there are over 2 million deaths related to physical inactivity each year. Furthermore, physical inactivity increases the risk of obesity which is one of the most significant health problems in the world. The WHO affirms that malnutrition is a health problem that is faced by many nations, and that one of the main reasons for malnutrition is that the consumption of energy-dense foods that are high in saturated fats and sugars, in combination with reduced physical activity.

The documentation of body mass index (BMI) and obesity are generally poor in primary care $[5,6]$. Obesity is associated with increased frequency of many longterm conditions that are import in both primary and secondary care, including type II diabetes, cardiovascular diseases and musculoskeletal problems [7]. Interactive Health Communication Applications (IHCAs) can support participatory decision making by providing the unique

\footnotetext{
$\dagger \quad$ Corresponding Author: Dept. of Computer Science and Engineering, Soonchunhyang University, Korea. (ynam@sch.ac.kr)

* Dept. of Civil \& Environmental Engineering, Worcester Polytechnic Institute, USA. (yeesock@wpi.edu)

Received: March 1, 2015; Accepted: September 1, 2015
}

infrastructure needed to deliver support for the multiple requirements of patients with chronic diseases (CDs) such as cardiovascular diseases, diabetes, cancer, and chronic respiratory diseases. IHCAs have also been used to facilitate patient adherence to weight loss regimens among overweight and obese women who were at an increased risk of developing breast cancer [8] and other chronic illnesses.

Obese people can manage and improve their health by following exercise and diet plans. For example, Mexico's social security institute recently presented PREVENIMSS [9], a program to prevent diseases caused by malnutrition and obesity. In this program, specialists assess the patient's health to recommend personalized diet and exercise plans, and patients are encouraged to manage and maintain their own unique plan. We believe that the development of an effective recommendation program with appropriate exercise and diet can be effective in treating and preventing obesity. Of particular interest are people who do not know what they should do to deal with obesity. With this in mind, and central to this research, is the development of an automatic recommendation system for exercise and diet such that effective nutrition and physical activity are maintained without intensive input from specialists. To achieve this goal, sensing methods have been developed that collect data including daily caloric expenditure, blood pressure, height, weight, total cholesterol, and blood sugar. Based on these collected data, the system described here provides appropriate recommendations for changes in exercise and diet.

The remainder of the paper is organized as follows. Previous work that is relevant to this study is discussed in Section 2. The architecture of the proposed system is then described in Section 3. Section 4 describes a performance evaluation via a 20 -day field testing. Concluding remarks 
are given in Section 5, and directions for future work are identified.

\section{Related Work}

There have been a number of systems developed to prevent and/or treat obesity, and researchers have considered the relationships between obesity, diet, and exercise. Triplebeat [10] estimates the heart rate and speed using processed sensed data, and assists users in achieving a predefined workout goal. The user interface consists of information including exercise goals, caloric expenditure, the position among a set of competitors, and heart rate. Click clique [11] is a preventative health care application that runs on a mobile phone. The application encourages teenage girls to exercise via interaction with their peers. MOPET [12] is a PDA-based personal trainer system that manages physical fitness activities. The system guides users through an exercise session, and provides advice based on current conditions. To solve obesity-related health problems, these systems encourage users to exercise more; however, the users should provide the goals matched to their requirements.

Other approaches to treat obesity include systems to encourage changes in diet and/or nutrition issues [13, 14]. Mankoff [14] designed an application to help users keep track of the nutritional content of food they eat. The system generates recommendations about healthier food items or supplementing missing nutrients using shopping receipts. Diet-aware Dining Table [13] is a dietary tracker system that uses a specialized dining table equipped with sensors. The dining table can track what is eaten and how many people; however, the system requires accurate information on the intake of a healthy and balanced diet, and the effectiveness of the system decreased when insufficient information was available. Although these systems exhibited meaningful results from a healthcare perspective, it is desirable to consider exercise as well as diet to improve the effectiveness of controlling obesity. Exercise without a healthy diet or a balanced diet without regular exercise are not sufficient; the system should recommend exercise and diet to users based on their personal health status.

Some researchers addressed the potential on the combination of the exercise and diet systems [14, 15], including Hyperfit [14], which provides an Internetbased service for personal management of nutrition and exercise. The service includes self-evaluation tools for goal definitions, diaries for food and exercise, testing and analysis tools, feedback, and recommendations from a virtual trainer. Silva's work [15] described a virtual specialist that stays with patients and gives users advice, such that the standard methods of diet and exercise are adhered to. However, it is difficult to manage personal health without various personal data using this system. With this in mind, in this study, we develop a new recommendation system that considers a balanced diet and appropriate exercise.

A behaviorally based, lifestyle, physical activity, counseling approach, in which sedentary individuals are encouraged to integrate more activity into their daily routines, by climbing stairs, taking short walks, and generally increasing daily activity, has been shown to be effective over a 2 -year period $[16,17]$. The number of global smartphone users surpassed 1 billion in 2014, and it is expected to reach 2 billion in 2015. Mobile phones have recently emerged as important devices for context-aware and intelligent services. Such high performance services have been achieved as a consequence of various embedded sensors, such as the Global Positioning System (GPS) and accelerometers; studies into human activity have been carried out using these built-in sensors, whereby various intelligent services were proposed using personal body motion data [18-21]. Here activity data were collected using a mobile device equipped with a three-axis accelerometer, and an intelligent system that recommends both exercise and diet suggestions was developed. Recently, the contributing factors to the obesity and physical inactivity epidemic in this population may be both diagnosis-specific and environmental [22]. Some studies have shown the beneficial effect of diet and physical activity on weight loss [23]. Wharton et al [24] found greater adherence to dietary tracking with a commercially available diet app (Lose It!).

\section{Recommendation System for Exercise and Diet}

The exercise and diet recommendation system described here is called SlimMe. It consists of four components: a sensor infrastructure, context management, a recommendation system and a display. The context management includes data on height, weight, blood sugar, total cholesterol, blood pressure, and amount of daily caloric expenditure. The recommendation system provides suggestions for appropriate exercise and diet based on the context data. The display is implemented as an intuitive graphical user

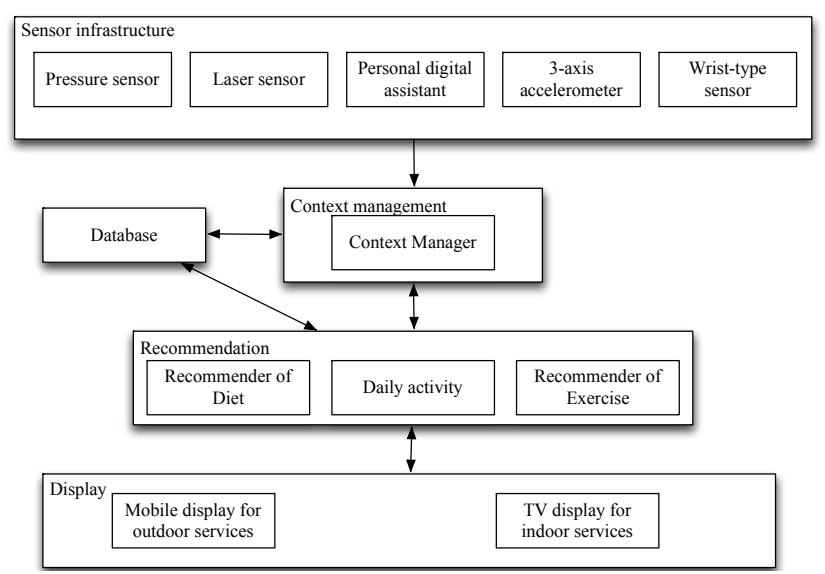

Fig. 1. System architecture of SlimMe 
interface (GUI). Fig. 1 shows the system architecture of SlimMe. In the context management, the context manager gathers and detects context information from the sensors. If the context is changed, the context manager notifies the adaptation manager and the observer component about the change. The main function of the context manager is to provide a mechanism for context binding between context entity (sensor infrastructure) and the observer component with a database.

\subsection{Sensor infrastructure}

To measure and record data on individuals, an area is designated as an entrance, and a pressure sensor and a laser are setup to determine weight and height, as shown in Fig. 2(a). Blood pressure is measured using a wrist-mounted sensor, as shown in Fig. 2(b). The total cholesterol and blood sugar are collected from electronic medical record (EMR) data and personal medical examinations using a mobile device, as these data are necessary to provide

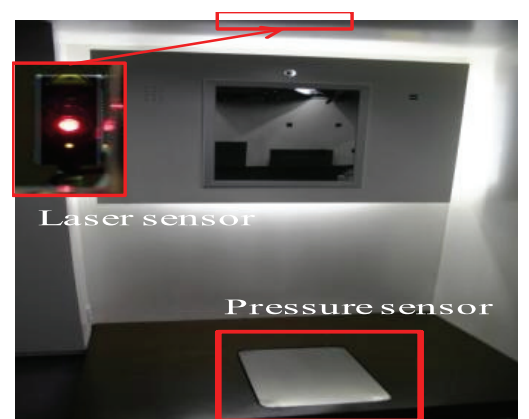

(a) pressure sensor and laser sensor

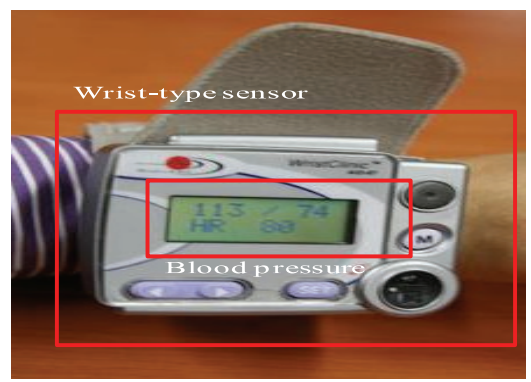

(b) wrist-type sensor

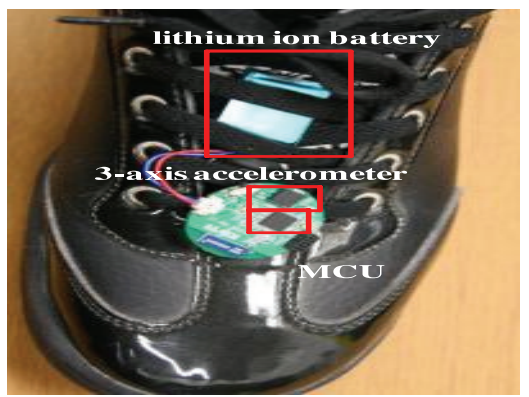

(c) 3-axis accelerometer

Fig. 2. Sensor infrastructure personal recommendations. To detect human activity, a smart shoe with a three-axis accelerometer and a microcontroller unit (MCU) was developed, as shown in Fig. 2(c).

\subsection{Context management}

The context manager collects contextual data from the sensors, the mobile device, and the smart shoe. The main function of the context manager is to convert these data into a suitable form, such as a daily caloric expenditure and BMI. The daily caloric expenditure was calculated as follows:

$$
E_{D C}=\alpha \cdot W \cdot T \cdot M E T S,
$$

where $E_{D C}$ is daily caloric expenditure [kcal], $\alpha$ is 0.0175 [kcal $/ \mathrm{kg} / \mathrm{min} / \mathrm{METs}], W$ is weight $[\mathrm{kg}] T$ is Time [min], and METs is METs value. $\alpha$ is calculated by $3.5 \mathrm{ml} / \mathrm{kg} / \mathrm{min}$. $0.005 \mathrm{kcal} / \mathrm{ml}$ [25].

The MET is a way of expressing the energy cost of physical activities as multiples of resting metabolic rate (RMR), and is defined as the ratio of

$$
\mathrm{BMI}=\frac{W}{H^{2}},
$$

where $W$ is weight $[\mathrm{kg}]$ and $H$ is height [m]. The recommenders can compare with the past context in database. In addition, the context manager transfers the processed context to the recommenders.

\subsection{Exercise and diet recommendation}

After collecting and analyzing the contextual data, the context manager transfers the processed data to the exercise and diet recommendation system. This system analyzes the data, and determines appropriate suggestions for exercise and diet. Here BMI is divided into five categories: normal, overweight, obese, super obese, and hyper obese. The exercise recommendations include four subcategories: walk slowly $(6 \mathrm{~km} / \mathrm{h})$, walk quickly $(8$ $\mathrm{km} / \mathrm{h})$, run slowly $(10 \mathrm{~km} / \mathrm{h})$, and run quickly $(12 \mathrm{~km} / \mathrm{h})$. To compute the default exercise recommendations, the system calculates the average of daily activity over the past 7 days. The default amount of exercise is the sum of this average, and the recommendations are based on BMI, and calculated using empirical data. From the average MET for activities described in [26], the exercise time can be calculated:

$$
\mathrm{T}_{E}=\frac{A_{R E}+A_{D A}}{\alpha \cdot W \cdot M E T s},
$$

where $A_{R E}$ is amount of recommended exercise based on 
BMI (kcal) and $A_{D A}$ is one week average of daily activity.

The daily routine of a person may vary from day to day, and the system should consider this to improve the reliability of the recommendations. The difference between the default level and the daily activity for the current day is calculated, and if this is negative, the system adds this to the default level of exercise for the following day. As listed in Table 1, a person (level 3,75 kg, one week of average daily activity; $132.5 \mathrm{kcal}$ ) needs to burn approximately $352.5 \mathrm{kcal}$ daily. If, for example, the daily activity for the current day is $120 \mathrm{kcal}$, the calculated exercise for the following day will be level 5 rather than level 3. As intensive exercise, the system recommends the maximum value for the category. Therefore, the exercise recommendation becomes $282.5 \mathrm{kcal}$.
The diet recommendation process is similar to that for exercise. The BMI is divided into categories as listed in Table 2. The system selects breakfast, lunch, or dinner as the primary classification, and the total cholesterol, blood sugar, and blood pressure as the secondary classification. The system then extracts the recommended diet to prevent hyperlipidemia, diabetes mellitus, and hypertension. A total cholesterol level of greater than $240 \mathrm{mg} / \mathrm{dl}$ is classified as hyperlipidemia. A blood sugar level above $110 \mathrm{mg} / \mathrm{dl}$ other than shortly after eating corresponds to diabetes mellitus. When the systolic blood pressure is consistently greater than $140 \mathrm{mmHg}$, this is considered hypertension. If the context data are outside of these levels, the system prohibits certain foods. Table 3 lists example diet recommendations, which include recommended meals,

Table 1. Sample data of recommended exercise based on five categories of BMI (weight: $75 \mathrm{~kg}$, one week average of daily activity: $132.5 \mathrm{kcal})$

\begin{tabular}{c|c|c|c|c|c|c|c}
\hline \multirow{2}{*}{ Level } & \multirow{2}{*}{ BMI } & \multirow{2}{*}{ Category } & \multicolumn{4}{|c|}{ Exercise time (min) } & ARE + \\
\cline { 4 - 8 } & & & Walk Slowly $(6 \mathrm{~km} / \mathrm{h})$ & Walk Quickly $(8 \mathrm{~km} / \mathrm{h})$ & Run Slowly $(10 \mathrm{~km} / \mathrm{h})$ & Run Quickly $(12 \mathrm{~km} / \mathrm{h})$ & ADA \\
\hline 5 & $18-22.9$ & Normal & 54 & 27 & 22 & 24 & 17 \\
\hline 4 & $23-24.9$ & Over Weight & 60 & 30 & 24 & 21 & 312.5 \\
\hline 3 & $25-29.9$ & Obese & 67 & 34 & 30 & 24 & 352.5 \\
\hline 2 & $30-39.9$ & Super Obese & 75 & 37 & 33 & 26 & 392.5 \\
\hline 1 & $40-$ & Hyper Obese & 82 & 41 & 24 & 432.5 \\
\hline
\end{tabular}

Table 2. Diet recommendation based on five categories of BMI

\begin{tabular}{|c|c|c|c|c|c|c|c|}
\hline \multirow{2}{*}{ Level } & \multirow{2}{*}{ BMI } & \multirow{2}{*}{ Category } & \multicolumn{4}{|c|}{ Total calories of recommended diet (kcal) } & \multirow{2}{*}{ Total } \\
\hline & & & Subcategory & Breakfast & Lunch & Dinner & \\
\hline \multirow{3}{*}{5} & \multirow{3}{*}{$18-22.9$} & \multirow{3}{*}{ Normal } & 1 & 481 & 682 & 763 & 1926 \\
\hline & & & 2 & 481 & 670 & 740 & 1891 \\
\hline & & & 3 & 411 & 510 & 626 & 1934 \\
\hline \multirow{3}{*}{4} & \multirow{3}{*}{$23-24.9$} & \multirow{3}{*}{ Over Weight } & 1 & 489 & 510 & 626 & 1625 \\
\hline & & & 2 & 444 & 510 & 650 & 1604 \\
\hline & & & 3 & 411 & 548 & 576 & 1535 \\
\hline \multirow{3}{*}{3} & \multirow{3}{*}{$25-29.9$} & \multirow{3}{*}{ Obese } & 1 & 363 & 495 & 539 & 1397 \\
\hline & & & 2 & 330 & 588 & 493 & 1411 \\
\hline & & & 3 & 412 & 472 & 468 & 1352 \\
\hline \multirow{3}{*}{2} & \multirow{3}{*}{$30-39.9$} & \multirow{3}{*}{ Super Obese } & 1 & 367 & 402 & 392 & 1161 \\
\hline & & & 2 & 370 & 406 & 399 & 1175 \\
\hline & & & 3 & 369 & 393 & 400 & 1162 \\
\hline \multirow{3}{*}{1} & \multirow{3}{*}{$40-$} & \multirow{3}{*}{ Hyper Obese } & 1 & 232 & 480 & 392 & 1104 \\
\hline & & & 2 & 219 & 406 & 398 & 1023 \\
\hline & & & 3 & 183 & 393 & 382 & 958 \\
\hline
\end{tabular}

Table 3. Example of meal recommendation

\begin{tabular}{|c|c|c|c|c|c|}
\hline BMI & Subcategory & Subcategory & Meal & Amount & Total Calories \\
\hline \multirow{12}{*}{3} & \multirow{12}{*}{ Menu2 } & \multirow{3}{*}{ Breakfast } & Cone flake & $1 / 2$ portion & \multirow{3}{*}{$330 \mathrm{kcal}$} \\
\hline & & & Milk & 1 glass & \\
\hline & & & Pear & 3 pieces & \\
\hline & & \multirow{4}{*}{ Lunch } & Fried-rice with ham and kimchi & 1 portion & \multirow{4}{*}{$588 \mathrm{kcal}$} \\
\hline & & & Fried eggs & 1 & \\
\hline & & & Radish kimchi & 1 portion & \\
\hline & & & Watermelon & 1 piece & \\
\hline & & \multirow{5}{*}{ Dinner } & Boiled barley and rice & 3/4 bowl & \multirow{5}{*}{$493 \mathrm{kcal}$} \\
\hline & & & Soybean paste soup & 3/4 bowl & \\
\hline & & & Braised hairtail fish & $3 / 4$ portion & \\
\hline & & & Braised burdock & $3 / 4$ portion & \\
\hline & & & Cabbage kimchi & $3 / 4$ portion & \\
\hline
\end{tabular}




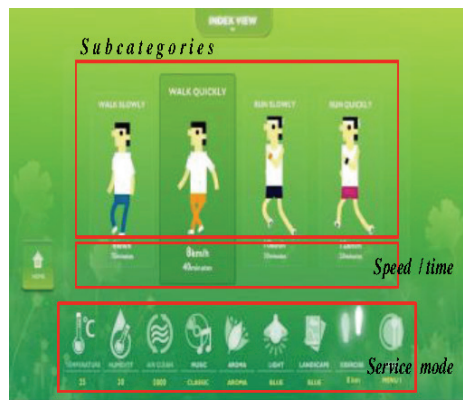

(a) UI for TV (exercise)

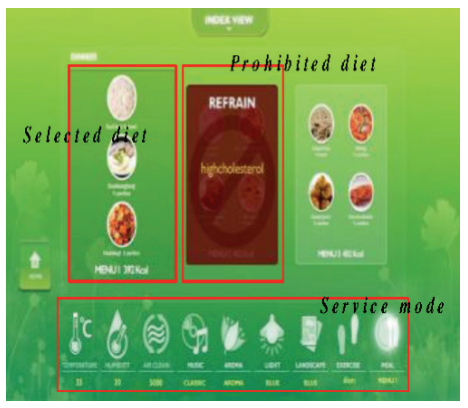

(b) UI for TV (diet)
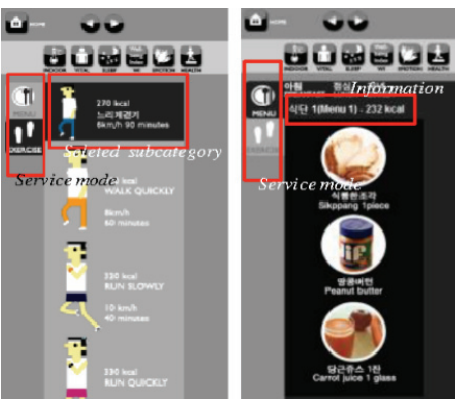

(c) UI for Mobile device (exercise and diet)

Fig. 3. Screenshots of user interfaces in SlimMe

portions, and the total caloric intake for subcategory 2 and level 3.

\subsection{Display}

To display the context data and recommendations, two display modes are proposed for indoor and outdoor services, as shown in Fig. 3. A user interface (UI) was developed for mobile phones using flash animations and 156 images. In addition, all operations are controlled using the touchscreen. After selecting a character image, customized information is sent to the treadmill in the home to select appropriate exercise without requiring manual setting, as shown in Fig. 3(a). In the diet recommendation screen, the system shows three sub-categories, with images showing information on menus, prohibited foods, and the total caloric intake, as shown in Fig. 3(b). The recommendations are stored in a database and transferred to the mobile device using a wireless network as shown in Fig. 3(c).

\section{Experimental Results}

\subsection{Experimental environments}

To evaluate SlimMe, we carried out at 20-day field trial, during which data were collected from three healthy participants, and diet and exercise recommendations were made. Table 4 lists the characteristics of the participants. The participants wore a WristClinic model AIO-67 sensor to measure blood pressure. A smart shoe was used to calculate the daily caloric expenditure. The smartphone was used to input the total cholesterol and blood sugar. The participants also used the mobile device for outdoor services. To calculate the daily caloric expenditure, activity

Table 4. Characteristics of participants

\begin{tabular}{c|c|c|c|c}
\hline Participant & Age & Gender & Initial BMI & Lifestyle \\
\hline A & 29 & Male & 25.77 & Stationary \\
\hline B & 25 & Female & 21.45 & Active \\
\hline C & 34 & Male & 25.83 & Stationary \\
\hline
\end{tabular}

was monitored from 9:00 am to 7:00 pm. To calculate the BMI, height and weight data were collected when the participants passed through a designated entrance.

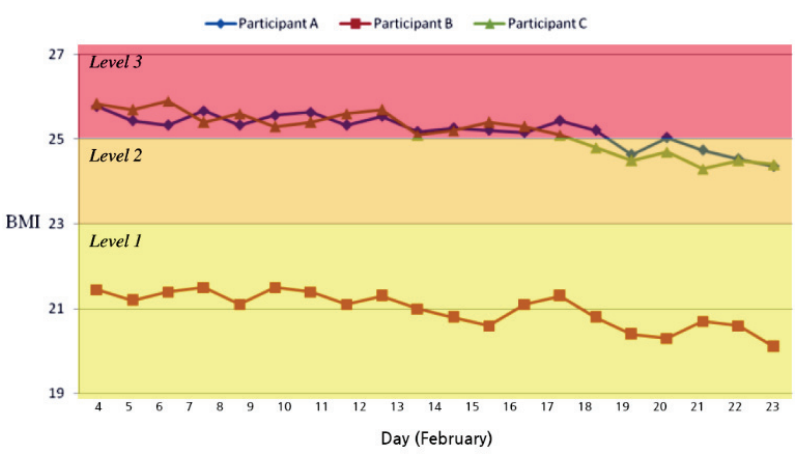

(a) Variation of BMI

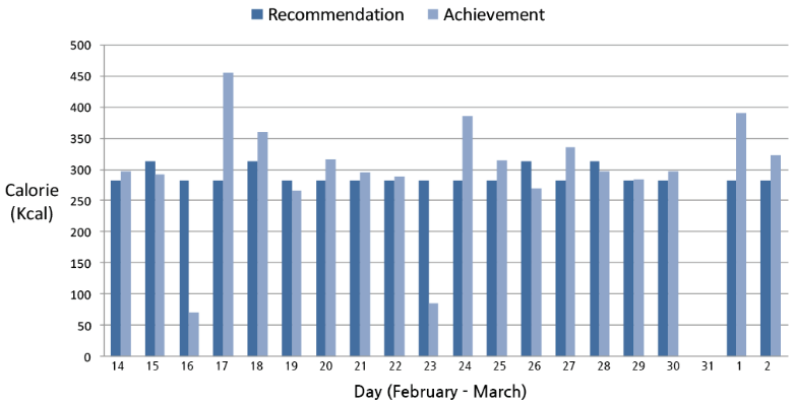

(b) Comparison between recommendation and achievement (participant A)

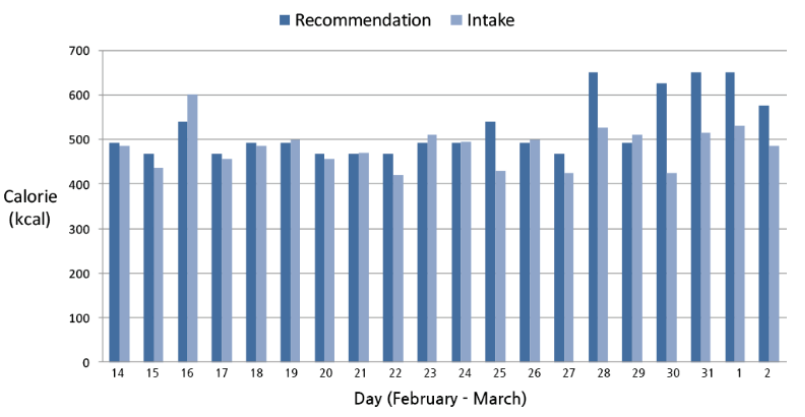

(c) Comparison between recommendation and intake (participant A)

Fig. 4. Experimental results about BMI, exercise, and diet 
Participants were asked to record their food consumption to calculate the caloric intake and to evaluate the usability of the system.

\subsection{Results}

The initial obesity level of the participants $\mathrm{A}$ and $\mathrm{C}$ was 3. After testing, both participants' levels dropped to level 2, which corresponds to overweight. This positive change demonstrates the usability of the system, as shown in Fig. 4(b). Figs. 4(b) and 4(c) show a comparison of the recommendations and the exercise and diet history. As shown in Fig. 4(b), participant A achieved the recommended value, surpassing it on most days; during weekends, participant A exceeded the exercise recommendations. The daily caloric expenditure on the 18th day was greater than the recommendation, so there was no diet recommendation, as shown in Fig. 4(b). Participant A also adhered to the diet recommendations, as shown in Fig. 4(c). Further details can be found in Appendix A.

\section{Conclusions and Discussions}

We have described an exercise and diet recommendation system, which can aid in the prevention and treatment of obesity by providing suggestions for exercise and diet, based on collected contextual health data. The system has display modes for both indoor and outdoor services. We carried out field tests, which showed that the system was effective in collecting and analyzing data, and that the recommendations were appropriate for both exercise and diet programs. After following the recommendations over the 20-day field test, the BMI of participants A and $\mathrm{C}$ fell from level 3 to level 2. The BMI of participant B also decreased. This result shows that the obesity of the participants was effectively managed via regular exercise and dietary changes over the short term. The two participants had sedentary lifestyles prior to the field test, and the recommendations for exercise, in particular, were challenging during the first few days; however, the change in BMI motivated the participants to follow the recommendations over the remainder of the field test.

Each of the factors addressed by the exercise and diet recommendation system may be significant in lifestyle changes. The system described here is a prototype, and there is a requirement for such systems to treat obesity. It is expected that the system will encourage patients to carry out regular exercise, and to follow diets that are healthy, thus changing their lifestyles to control obesity. As part of future work, we plan to focus on improving the performance of the system through extensive field tests. In the near future, we plan to evaluate the usability of the current prototype system over one month via field tests with a larger number of participants.

\section{Acknowledgements}

This work was supported by the Soonchunhyang University Research Fund and was also supported by the MSIP (Ministry of Science, ICT and Future Planning), Korea, under the C-ITRC(Convergence Information Technology Research Center) (IITP-2015-H8601-15-1009) supervised by the IITP(Institute for Information \& communications Technology Promotion)

\section{References}

[1] World Health Organisation, "Obesity: preventing and managing the global epidemic," Report of a WHO consultation. Tech. rep., WHO, Geneva (2000)

[2] A.A. Hedley, et al. "Prevalence of overweight and obesity among US children, adolescents, and adults, 1999-2002," JAMA: the journal of the American Medical Association, vol. 291, no. 23, pp. 2847-2850, 2004.

[3] K.F. Ferraro, et al. "The life course of severe obesity: does childhood overweight matter?" The Journals of Gerontology Series B: Psychological Sciences and Social Sciences, vol. 58, no. 2, pp. S110-S119, 2003.

[4] Office of the Surgeon General. "The Surgeon General's call to action to prevent and decrease overweight and obesity," Public Health Service, 2001.

[5] H.P. Booth, et al. "Epidemiology of clinical body mass index recording in an obese population in primary care: a cohort study," Journal of Public Health, vol. 35, pp. 67-74, 2013.

[6] M.E. Waring, et al. "Documentation and management of overweight and obesity in primary care," The Journal of the American Board of Family Medicine, vol. 22. No.5, pp. 544-552, 2009.

[7] A. Must, et al. "The disease burden associated with overweight and obesity," Jama, vol. 282, no. 16, pp. 1523-1529, 1999.

[8] L. Cadmus-Bertram, et al. "Web-based self-monitoring for weight loss among overweight/obese women at increased risk for breast cancer: the HELP pilot study," Psycho-Oncology, vol. 22, no. 8, pp. 18211828, 2013.

[9] G. Gutiérrez, et al. "Strengthening preventive care programs: a permanent challenge for healthcare systems; lessons from PREVENIMSS México," BMC public health vol. 10, no. 1, pp. 417, 2010.

[10] R. de Oliveira, et al. "TripleBeat: enhancing exercise performance with persuasion," in Proceedings of the 10th international conference on Human computer interaction with mobile devices and services, pp. 255264, 2008.

[11] T. Toscos, et al. "Chick clique: persuasive technology to motivate teenage girls to exercise," in CHI'06 extended abstracts on Human factors in computing 
systems, pp. 1873-1878, 2006.

[12] F. Buttussi, L. Chittaro, "A context-aware and useradaptive wearable system for fitness training," Artificial Intelligence in Medicine, vol. 42, no. 2, pp. 153-163, 2008.

[13] K.h. Chang, et al. "The diet-aware dining table: Observing dietary behaviors over a tabletop surface," in Pervasive computing, pp. 366-382, 2006.

[14] J. Mankoff, et al. "Using low-cost sensing to support nutritional awareness," in UbiComp 2002: Ubiquitous Computing, pp. 371-378, 2002.

[15] J.M. Silva, et al. "Promoting a healthy lifestyle through a virtual specialist solution," in CHI'06 Extended Abstracts on Human Factors in Computing Systems, pp. 1867-1872. 2006

[16] Andrea L. Dunn, et al. "Lifestyle physical activity interventions: history, short-and long-term effects, and recommendations," American journal of preventive medicine, vol. 15, no.4, pp. 398-412, 1998.

[17] Andrea L. Dunn, et al. "Comparison of lifestyle and structured interventions to increase physical activity and cardiorespiratory fitness: a randomized trial," Jama, vol. 281, no.4, pp. 327-334, 1999.

[18] A. G. Bonomi, and K. R. Westerterp, "Advances in physical activity monitoring and lifestyle interventions in obesity: a review," International Journal of Obesity, vol. 36, no. 2, pp. 167-177, 2012.

[19] Eugene Oteng-Ntim, et al. "Lifestyle interventions for overweight and obese pregnant women to improve pregnancy outcome: systematic review and meta-analysis," BMC medicine, vol. 10, no. 1, pp. 47, 2012.

[20] Anne R. Lindsay, et al. "Field Assessments for obesity prevention in children and adults: physical activity, fitness, and body composition," Journal of nutrition education and behavior, vol. 46, no.1, pp. 43-53, 2014.

[21] Wener Hoeger, and Sharon Hoeger, "Lifetime physical fitness and wellness: A personalized program," Cengage Learning, 2014.

[22] Sudha M. Srinivasan, et al. "Current perspectives on physical activity and exercise recommendations for children and adolescents with autism spectrum disorders," Physical therapy, 2014.

[22] Alicia S. Kunin-Batson, et al. "Household Factors, Family Behavior Patterns, and Adherence to Dietary and Physical Activity Guidelines Among Children at Risk for Obesity," Journal of nutrition education and behavior, vol. 47, no. 3, pp. 206-215, 2015.

[23] N. Travier, et al. "Effect of a diet and physical activity intervention on body weight and nutritional patterns in overweight and obese breast cancer survivors. Medical Oncology," vol. 31, no.1, pp. 1-11, 2014.

[24] Christopher M. Wharton, et al. "Dietary selfmonitoring, but not dietary quality, improves with use of smartphone app technology in an 8-week weight loss trial," Journal of nutrition education and behavior, vol. 46, no. 5, pp. 440-444, 2014.

[25] Somdat Mahabir, et al. "Comparison of energy expenditure estimates from 4 physical activity questionnaires with doubly labeled water estimates in postmenopausal women," The American journal of clinical nutrition, vol. 84, no.1, pp. 230-236, 2006.

[26] G.A. Chase, Average met values for activities with fitness components (2015), http://community.plu.edu/ chasega/main.htm.

[27] Medic4all, Medic4all's Wrist Clinic (2015), http:// en.medic4all.it/products-and-tecnology/products.

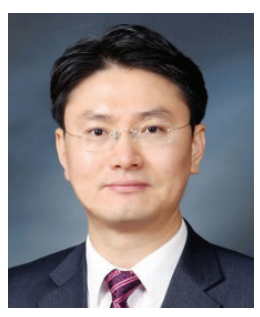

Yunyoung Nam He received the B.S., M.S., and Ph.D. degrees in computer engineering from Ajou University, Korea in 2001, 2003, and 2007 respectively. He was a Senior Researcher in the Center of Excellence in Ubiquitous System (CUS) from 2007 to 2010 . He was a Research Professor in Ajou University from 2010 to 2011 . He also spent time as a Visiting Scholar at Center of Excellence for Wireless \& Information Technology (CEWIT), Stony Brook University, New York. He was a Postdoctoral Fellow at Worcester Polytechnic Institute, Massachusetts from 2013 to 2014. $\mathrm{He}$ is currently an assistant professor in the Department of Computer Science and Engineering at Soonchunhyang University. His research interests include multimedia database, ubiquitous computing, image processing, pattern recognition, context-awareness, wearable computing, intelligent video surveillance, cloud computing, and biomedical signal processing.

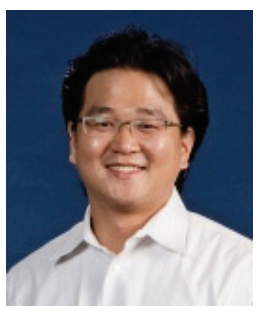

Yeesock Kim He received his B.S., M.S. and Ph.D. degrees in structural engineering from Catholic Kwandong Univ. in 2000, Yonsei Univ. in 2002, and Texas A\&M Univ. in 2007, respectively, then completed postdoctoral training in control engineering at Texas A\&M Univ. in 2010. Since 2010, he has been an assistant professor of structural engineering at Worcester Polytechnic Institute in USA. His research interests are signal processing, machine learning, and control system design. 


\section{Appendix}

Data log examples of exercise and diet recommendations

Table 5. Diet recommendation based on five categories of BMI

\begin{tabular}{|c|c|c|c|c|c|c|}
\hline Date & $\begin{array}{l}\text { Daily } \\
\text { activity } \\
\text { (kcal) }\end{array}$ & $\begin{array}{l}\text { Calorie of default } \\
\text { recommended } \\
\text { exercise(kcal) }\end{array}$ & $\begin{array}{c}\text { Calorie of real } \\
\text { recommended exercise/ } \\
\text { achievement(kcal) }\end{array}$ & $\begin{array}{l}\text { Calorie of recommended } \\
\text { diet/ Prohibited diet }\end{array}$ & Intake & Summary of day \\
\hline 2014-02-04(Tue) & 136.7 & 352.5 & $282.5 / 300$ & 493(dinner) / No & $485 \mathrm{kcal}$ & normal /indoor exercise \\
\hline 2014-02-05(Wen) & 69 & 352.5 & $312.5 / 292$ & 468(dinner) / No & $435 \mathrm{kcal}$ & normal/indoor exercise \\
\hline 2014-02-06(Thu) & 102 & 352.5 & $282.5 / 71$ & $539 /$ Yes & $600 \mathrm{kcal}$ & drinking \\
\hline 2014-02-07(Fri) & 317 & 352.5 & 282.5/455-outdoor & $\begin{array}{c}\text { 495(lunch), } \\
468 \text { (dinner)/ No }\end{array}$ & $485 / 455 \mathrm{kcal}$ & $\begin{array}{c}\text { shopping/ } \\
\text { outdoor exercise }\end{array}$ \\
\hline 2014-02-08(Sat) & 52 & 352.5 & 312.5/359-outdoor & $\begin{array}{c}\text { 588(lunch), } \\
\text { 493(dinner)/ No }\end{array}$ & $535 / 485 \mathrm{kcal}$ & normal/ outdoor exercise \\
\hline 2014-02-09(Sun) & 134 & 352.5 & $282.5 / 266$ & 493(dinner) / No & $500 \mathrm{kcal}$ & normal /indoor exercise \\
\hline 2014-02-10(Mon) & 117 & 352.5 & $282.5 / 316$ & 468(dinner) /No & $455 \mathrm{kcal}$ & normal /indoor exercise \\
\hline 2014-02-11(Tue) & 98 & 352.5 & $282.5 / 295$ & 468(dinner) /No & $470 \mathrm{kcal}$ & normal /indoor exercise \\
\hline 2014-02-12(Wed) & 102 & 352.5 & $282.5 / 288$ & 468(dinner) /No & $420 \mathrm{kcal}$ & normal /indoor exercise \\
\hline 2014-02-13(Thu) & 119 & 352.5 & $282.5 / 86$ & 493(dinner) / No & $510 \mathrm{kcal}$ & normal /indoor exercise \\
\hline 2014-02-14(Fri) & 348 & 352.5 & 282.5/386-outdoor & $\begin{array}{c}\text { 472(lunch), } \\
\text { 493(dinner)/ No }\end{array}$ & $455 / 495 \mathrm{kcal}$ & normal/outdoor exercise \\
\hline 2014-02-15(Sat) & 288 & 352.5 & 282.5/315-outdoor & $\begin{array}{c}\text { 495(lunch), } \\
539 \text { (dinner)/ No }\end{array}$ & $525 / 430 \mathrm{kcal}$ & normal/outdoor exercise \\
\hline 2014-02-16(Sun) & 57 & 352.5 & $312.5 / 47$ & 493(dinner) / No & $500 \mathrm{kcal}$ & normal /indoor exercise \\
\hline 2014-02-17(Mon) & 101 & 352.5 & $282.5 / 335$ & 468(dinner) /No & $425 \mathrm{kcal}$ & normal /indoor exercise \\
\hline 2014-02-18(Tue) & 42 & 352.5 & $312.5 / 297$ & 650 (dinner) / No & $475 \mathrm{kcal}$ & normal /indoor exercise \\
\hline 2014-02-19(Wed) & 122 & 312.5 & $282.5 / 284$ & 493(dinner) / No & $510 \mathrm{kcal}$ & normal /indoor exercise \\
\hline 2014-02-20(Thu) & 83 & 352.5 & $282.5 / 296$ & 626(dinner) / No & $425 \mathrm{kcal}$ & normal /indoor exercise \\
\hline 2014-02-21(Fri) & 402 & 312.5 & commendation & $\begin{array}{c}548(\text { lunch }), \\
650 \text { (dinner)/ No }\end{array}$ & $550 / 415 \mathrm{kcal}$ & outdoor exercise \\
\hline 2014-02-22(Sat) & 227 & 312.5 & $282.5 / 390$ - outdoor & 650 (dinner) / No & $430 \mathrm{kcal}$ & normal /outdoor exercise \\
\hline 2014-02-23(Sun) & 97 & 312.5 & $282.5 / 322$ & 576(dinner)/ No & $385 \mathrm{kcal}$ & normal/indoor exercise \\
\hline
\end{tabular}

